\title{
The burden of low back pain, rheumatoid arthritis, osteoarthritis, and gout and their respective attributable risk factors in Brazil: results of the GBD 2017 study
}

\author{
Juliana Wolf ${ }^{11,[2], ~ E l i s a b e t h ~ B a r b o z a ~ F r a n c ̧ a ~}{ }^{[1]}$ and Ada Ávila Assunçãoo ${ }^{[1],[2]}$
}

[1]. Universidade Federal de Minas Gerais, Faculdade de Medicina, Programa de Pós-Graduação em Saúde Pública, Belo Horizonte, MG, Brasil. [2]. Universidade Federal de Minas Gerais, Núcleo de Estudos Saúde e Trabalho, Belo Horizonte, MG, Brasil.

\begin{abstract}
Introduction: Musculoskeletal (MSK) disorders are a major cause of disability worldwide. Different modifiable risk factors are associated to these disorders. The objective of this study was to analyze the burden of low back pain (LBP), rheumatoid arthritis (RA), osteoarthritis (OA), and gout, attributable to risk factors, in 2017. Methods: The burden of LBP, RA, OA, and gout, and attributable risk factors were analyzed using data extracted from the Global Burden of Disease (GBD) Brasil-2017 study. Descriptive analysis was conducted to compare disability-adjusted life years (DALY) rates between sexes and age groups (15-49 and 50-69 years), in 2017. Results: The highest rates of DALY due to LBP were attributed to occupational ergonomic factors in the 15-49-year group, regardless of sex and males aged 50-69 years, whereas smoking was the major contributor in the 50-69-year female group. RA-related DALY rates were attributed to smoking and were higher among women aged 50-69 years. High body mass index (BMI) was the most relevant risk factor for the burden of OA, with higher rates detected in the 50-69-year group, and it was the most significant risk factor for DALY rate attributed to gout, regardless of sex or age group. Conclusions: Occupational surveillance measures are indicated to prevent LBP. Actions to decrease smoking and overweight, and the surveillance of weight gain are warranted to decrease the burden of LBP, RA and $\mathrm{OA}$, and gout, respectively. These actions will be more effective if age and sex differentials are considered in planning.
\end{abstract}

Keywords: Low back pain. Rheumatoid arthritis. Osteoarthritis. Gout. Risk factors. Global Burden of Disease.

\section{INTRODUCTION}

Musculoskeletal (MSK) disorders occur as a result of inflammatory or degenerative processes affecting musculoskeletal tissues. Different regions of the human body may be affected, such as the cervical and lumbar regions, and the upper and lower limbs ${ }^{1}$. Pain causes muscle stiffness and reduced mobility, leading to dependence, disability, and deformity ${ }^{2}$. Social interaction impairment, low levels of well-being and compromised workrelated functions are dimensions of the impact of this disease $\mathrm{e}^{3,4}$.

These disorders were the primary cause of duty-related disability retirement in 2017 (15,522 men and 14,972 women) in Brazilian urban areas, accounting for $17.4 \%$ of all retirements, and amounting to 45.5 million Brazilian reals in social security retirement benefits.

\footnotetext{
Corresponding author: Juliana Wolf.

e-mail: julianawolf09@gmail.com

(D) https://orcid.org/0000-0002-7284-7169

Received 4 May 2021

Accepted 9 September 2021
}

The number of paid sick leaves granted to eligible urban and rural residents totaled up 329,347 and 37,435 , respectively ${ }^{5}$.

In the Global Burden of Disease (GBD) study, MSK disorders comprise all musculoskeletal system diseases, which are further divided into six subcategories: low back pain (LBP), rheumatoid arthritis (RA), osteoarthritis (OA), gout, neck pain (NP) and "others", the latter including remaining MSK disorders subcategories ${ }^{6}$. The global incidence of MSK disorders increased between 1990 and 2017. However, the age-standardized incidence rate of these disorders has been declining 7 .

Disability-adjusted life years (DALY) is a useful metric to determine the impact of MSK disorders and other morbidities in public health. DALY is a summary measure of the GBD study, which combines years of life lost (YLL) due to premature death and years lived with disability (YLD) ${ }^{6}$.

MSK disorders, major cause of sickness absence worldwide, went from ranking $10^{\text {th }}$, in 2000 , to $5^{\text {th }}$, in 2015 , among 23 ICD-10 categories ${ }^{8}$. Data on the prevalence of risk factors have been provided in studies conducted with samples comprising 
specific professional categories ${ }^{9-11}$. However, studies investigating the Brazilian adult population are scarce ${ }^{12}$.

In this study, we used data extracted from the GBD 2017 study to analyze the burden of LBP, RA, OA, and gout, attributable to risk factors according to sex and age group, in 2017.

\section{METHODS}

\section{Study design and current analysis}

This descriptive study was based on estimates of the GBD study, 2017. Data in that study were provided by the Institute for Health Metrics and Evaluation (IHME), from Washington University, Unites States (USA) ${ }^{13}$, and indicators were calculated and updated in the corresponding website ${ }^{14}$.

The GBD 2017 study includes estimates for 359 diseases and injuries, 3484 sequelae and 84 risk factors, for 195 countries and territories. LBP, RA, OA, and gout are classified with the following codes according to the International Classification of Diseases (ICD-10): LBP (M54.3, M54.4 and M54.5), RA (M05, M06 and M08), OA (M16 and M17), gout (M10), NP (M54.2) and other MSK (L93, M00-M02, M08, M11-M13, M20-M25, M30-M35, M40-M43, M45-M46, M60 -M63, M65-M68, M70- M73, M75-M79, M80-M85, M86, M87-M90, M91-M94, M95-M99)6. Risk factors are grouped into three categories: behavioral, metabolic, and environmental/occupational comprising four levels each $^{15}$. In the GBD study, the burden of MSK disorders and risk factors was estimated from Brazilian data obtained from several sources, including census, hospital records, national surveys, technical reports, and the scientific literature ${ }^{16}$.

In this study, data were analyzed by sex and age group (15-49 and 50-69 years) for 2017. The estimates were presented in proportions, absolute number, and rates per 100,000 and their respective $95 \%$ uncertainty interval (UI) ${ }^{17}$. NP and "other MSK disorders" were not associated with risk factors examined in the GBD study. Therefore, these causes were not included in this analysis. All level 3 (occupational ergonomic factors, smoking, high body mass index (BMI), and impaired kidney function) risk factors as per the GBD study were accounted for. These risk factors meet the convincing or probable evidence of causation criteria established by the GBD 2017 study $^{15}$.

Occupational ergonomic factors were defined as the proportion of the working population exposed to low back pain-inducing work, based on population distributions across nine occupational categories, and the Theoretical Minimum Risk Exposure Level (TMREL) was assumed to be no exposure to that risk. Smoking was defined as current and former smoking of any tobacco product, and TMREL was zero smoking. High BMI was considered as BMI greater than 20 to $25 \mathrm{~kg} / \mathrm{m}^{2}$ and the TMREL was determined based on the BMI level, which was associated with the lowest risk of all-cause mortality in prospective cohort studies. Impaired kidney function risk factor exposure was defined by urinary albumin to creatinine ratio (ACR) and estimated glomerular filtration rate (eGFR): albuminuria with preserved GFR, chronic kidney disease (CKD) stage 3, 4 and 5 . The TMREL for impaired kidney function was a diagnosis of albuminuria or CKD stages 3,4 , or $5^{15}$.
This study utilized only secondary databases which are publicly available, while respecting the ethical principles of Resolution no. 466/2012 of the Brazilian Conselho Nacional de Saúde. The GBD Brazil 2015 project was approved by the Research Ethics Committee of Universidade Federal de Minas Gerais, CAAE 62803316.7.0000.5149.

\section{GBD Study variables}

For a given population, DALY are calculated as the sum of YLL due to premature death, and YLD, per age group, sex, location, and cause. The YLL is calculated as the estimated number of deaths at a particular age multiplied by the standard life expectancy at the age at which death occurs. The YLD is calculated as the prevalence of the disease multiplied by a weight factor attributed to each type of disability. Disability weights reflect the magnitude of health loss due to a disease, measured on a zero to one scale, where zero indicates full health and one is equivalent to death. The input data for all MSK disorders were obtained through scientific literature, surveys and vital registration. Adjustments were applied to extracted data to make it more consistent and suitable for modelling. Mortality was estimated using the Cause of Death Ensemble model (CODEm). This analytic tool generates a combined set of models for enhanced predictive performance regarding cause-specific mortality estimates ${ }^{18}$. Morbidity was estimated using the modelling system DisMod-MR 2.1 ${ }^{6}$. Further methodological details are available $e^{6,18,19}$.

The burden attributable to risk factors expresses a hypothetic reduction in the current disease burden, which would be expected if an alternative or counterfactual distribution of previous exposure had been applied. Attributable burden estimates take into account four key components: burden quantification metric, levels of exposure to a risk factor, relative risk of a given exposure-related effect, and counterfactual risk factor exposure level. The TMREL concept was employed in the GBD study. Estimates of attributable burden, such as DALY for risk-outcome pairs, were calculated as the DALY multiplied by the population attributable fraction (PAF) for a risk-outcome pair for a given age, sex, location, and year. In short, PAF is the proportional risk reduction that would occur in a given year if previous exposure to a risk factor were reduced to the counterfactual level ${ }^{15}$. Detailed description of this methodology is available in published GBD studies ${ }^{15}$.

\section{RESULTS}

In 2017, LBP was the major contributor to DALY due to MSK disorders among Brazilian men and women in both age groups considered, followed by other MSK disorders and NP. The proportional contribution of LBP was higher in the 15-49-year group $(69.58 \%$ and $62.02 \%$, men and women, respectively) compared to 50-69-year group, while NP, OA, RA, and gout prevailed in the 50-69-year group when compared to 15-49-year group (Table 1).

The proportion of DALY that could be attributed to the risk factors currently assessed in GBD 2017, ranged from 52.89\% for gout to $0 \%$ for NP and other MSK disorders (Figure 1). No association between risk factors and NP and other MSK disorders was identified. 
TABLE 1: Contribution of LBP, NP, RA, OA, gout and other MSK disorders to the total DALY caused by MSK disorders, by sex and age group. Brazil, 2017.

\begin{tabular}{|c|c|c|c|c|c|c|c|c|c|c|c|c|}
\hline \multirow{3}{*}{ MSK disorders } & \multicolumn{6}{|c|}{ Men } & \multicolumn{6}{|c|}{ Women } \\
\hline & \multicolumn{3}{|c|}{15 to 49 years } & \multicolumn{3}{|c|}{50 to 69 years } & \multicolumn{3}{|c|}{15 to 49 years } & \multicolumn{3}{|c|}{50 to 69 years } \\
\hline & Number & $\%$ & Rate* $^{*}$ & Number & $\%$ & Rate* $^{*}$ & Number & $\%$ & Rate* $^{*}$ & Number & $\%$ & Rate* $^{*}$ \\
\hline LBP & $808,481.18$ & 69.6 & $1,432.53$ & $396,333.67$ & 57.5 & $2,207.16$ & $797,216.82$ & 62.0 & $1,376.49$ & $440,896.14$ & 53.5 & $2,174.62$ \\
\hline NP & $133,559.81$ & 11.5 & 236.65 & $96,641.43$ & 14.0 & 538.19 & $155,525.35$ & 12.1 & 268.53 & $122,477.81$ & 14.9 & 604.09 \\
\hline OA & $19,968.94$ & 1.7 & 35.38 & $55,470.57$ & 8.0 & 308.91 & $21,042.11$ & 1.6 & 36.33 & $62,842.04$ & 7.6 & 309.95 \\
\hline RA & $13,023.77$ & 1.1 & 23.08 & $13,843.34$ & 2.0 & 77.09 & $24,046.91$ & 1.9 & 41.52 & $30,978.69$ & 3.8 & 152.80 \\
\hline Gout & $5,178.56$ & 0.4 & 9.18 & $5,845.02$ & 0.8 & 32.55 & $25,78.93$ & 0.2 & 4.45 & $3,529.85$ & 0.4 & 17.41 \\
\hline Other MSK disorders & $181,727.32$ & 15.6 & 322.00 & $121,273.83$ & 17.6 & 675.37 & $284,994.68$ & 22.2 & 492.08 & $162,662.82$ & 19.8 & 802.30 \\
\hline
\end{tabular}

${ }^{*}$ Rate per 100,000. LBP: low back pain; NP: neck pain; RA: rheumatoid arthritis; OA: osteoarthritis; MSK: Musculoskeletal; DALY: Disability-adjusted life years.

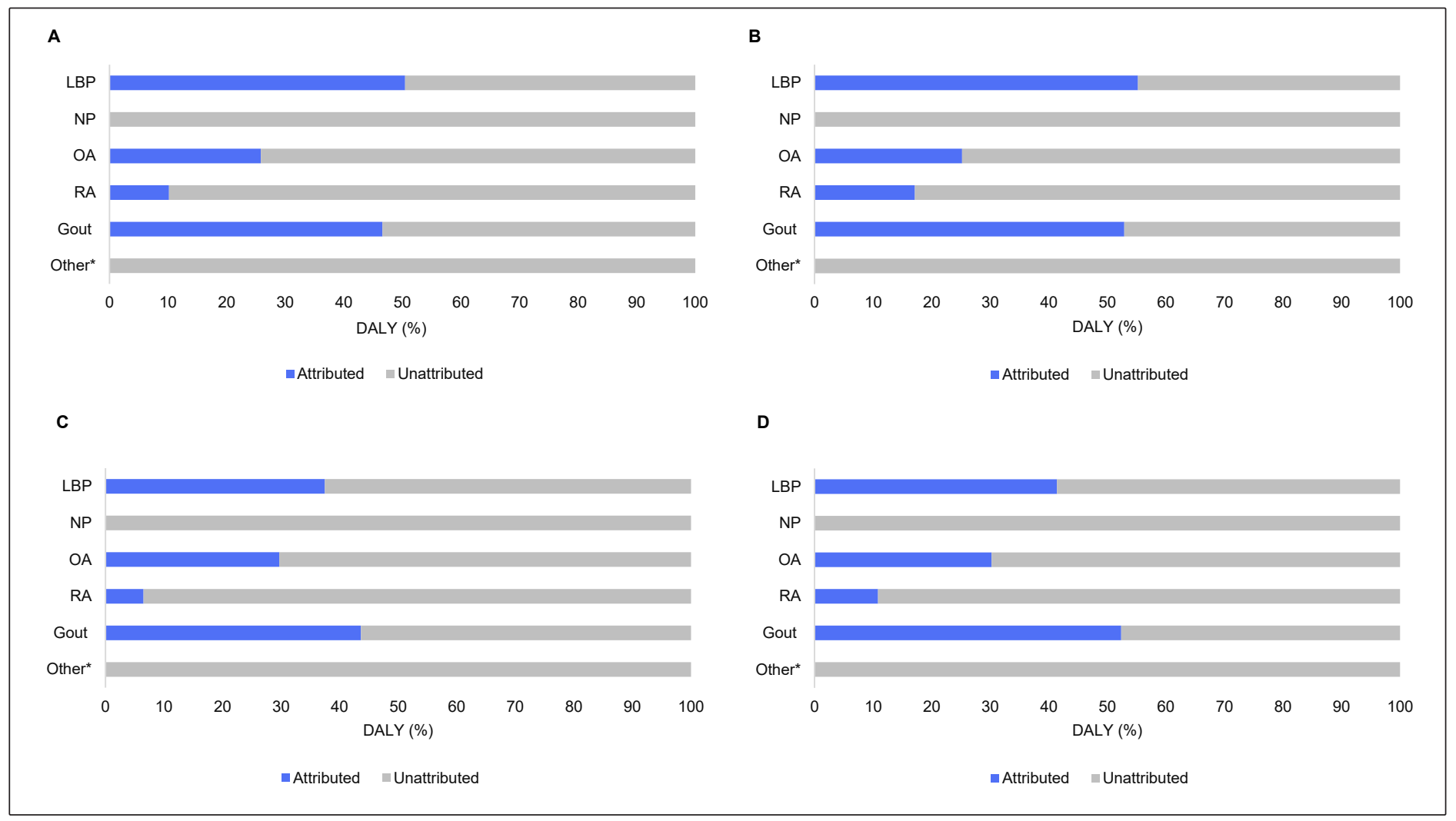

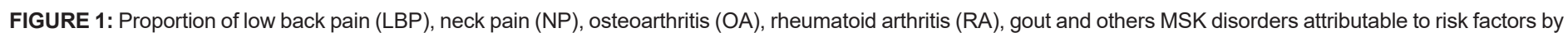

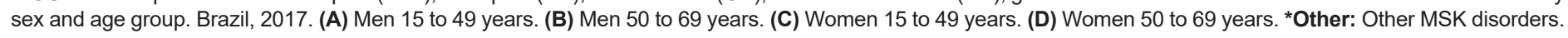

Occupational ergonomic risk was the risk factor that most contributed to the LBP burden among men (535.33 per 100,000) and women $(349.88$ per 100,000$)$ aged $15-49$ years. For women aged 50-69 years, smoking $(446.73$ per 100,000) was the main risk factor for LBP (Figure 2, Table 2).

The overall burden of RA was attributed to smoking. DALY rates were higher in the 50-69-year group among women $(16.54$ per 100,000). High BMI was the primary risk factor for the burden of OA. The rate of OA-related DALY attributed to high BMI was higher among women in both age groups, particularly 50-69 years (93.76 per 100,000) (Figure 2, Table 2).
High BMI was also a major contributor to the gout burden attributable to risk factors in men and women, in both age groups. The rate of DALY due to gout attributable to high BMI was higher among men aged 50-69 years (14.77 per 100,000) (Figure 2, Table 2).

\section{DISCUSSION}

GBD 2017 showed LBP as the main cause of DALY among all MSK disorders, and it is mainly attributed to ergonomic risk factors. DALY related to RA, OA, and gout were mainly attributed to behavioral risk factors, including smoking for the RA and BMI for the other two. Regarding NP, it was not possible to describe the 
Wolf $\mathrm{J}$ et al. - The burden of low back pain, rheumatoid arthritis, osteoarthritis, gout, and risk factors in Brazil

TABLE 2: DALY rate and their respective $95 \%$ uncertainty interval (UI) caused by LBP, RA, OA, and gout attributable to level 3 risk factors, by sex and age group, per 100,000. Brazil, 2017.

\begin{tabular}{|c|c|c|c|c|c|c|c|c|c|}
\hline \multirow{3}{*}{ Cause } & \multirow{3}{*}{ Risk factors } & \multicolumn{4}{|c|}{ Men } & \multicolumn{4}{|c|}{ Women } \\
\hline & & \multicolumn{2}{|c|}{15 to 49 years } & \multicolumn{2}{|c|}{50 to 69 years } & \multicolumn{2}{|c|}{15 to 49 years } & \multicolumn{2}{|c|}{50 to 69 years } \\
\hline & & Rate & UI 95\% & Rate & UI $95 \%$ & Rate & UI $95 \%$ & Rate & UI 95\% \\
\hline \multirow{3}{*}{ LBP } & Occupational ergonomic factors & 535.33 & $367.77-755.14$ & 703.15 & $457.21-1015.77$ & 349.88 & $239.10-497.92$ & 378.63 & $247.72-551.05$ \\
\hline & Smoking & 216.69 & $133.88-320.63$ & 603.37 & $373.38-900.21$ & 131.50 & 81.3-194.34 & 446.73 & $278.69-669.12$ \\
\hline & High BMI & 111.46 & $58.48-185.95$ & 208.91 & $110.94-369.07$ & 109.75 & $62.2-174.55$ & 234.65 & $128.13-395.95$ \\
\hline RA & Smoking & 2.34 & $0.61-4.63$ & 13.19 & $3.80-25.60$ & 2.70 & $0.60-5.54$ & 16.54 & $3.97-32.66$ \\
\hline $\mathrm{OA}$ & High BMI & 9.15 & $3.35-19.62$ & 77.81 & $29.44-174.13$ & 10.79 & $4.19-23.61$ & 93.76 & $36.08-205.10$ \\
\hline \multirow{2}{*}{ Gout } & High BMI & 4.10 & $1.94-7.40$ & 14.77 & $7.12-25.46$ & 1.83 & $0.97-2.98$ & 7.49 & $3.95-12.04$ \\
\hline & Impaired kidney function & 0.31 & $0.19-0.48$ & 4.45 & $2.83-6.38$ & 0.20 & $0.12-0.31$ & 2.86 & $1.81-4.12$ \\
\hline
\end{tabular}

DALY: disability-adjusted life years; LBP: low back pain; RA: rheumatoid arthritis; OA: osteoarthritis; UI: Uncertainty Interval.

\section{A}

Occupational ergonomic

Smoking

High BMI

Impaired kidney function

Others* $0 \quad 100$ $200 \quad 300 \quad 400 \quad 500$
DALY per 100,000

c

Occupational ergonomic

$\varpi \mathrm{LBP} \backsim \mathrm{RA} \backsim \mathrm{OA} \backsim$ Gout

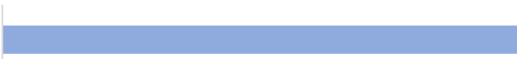

0
Smoking

High BMI

Impaired kidney function

Others*

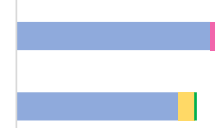

DALY per 100,000

$\square \mathrm{LBP} \backsim \mathrm{RA} \backsim \mathrm{OA} \backsim$ Gout
B

Occupational ergonomic

Smoking

Smoking

High BMI

|

Impaired kidney function

Others*

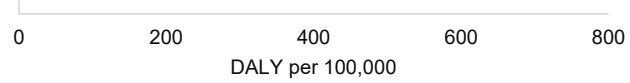

$\because \mathrm{LBP} \backsim \mathrm{RA} \backsim \mathrm{OA} \backsim$ Gout

D

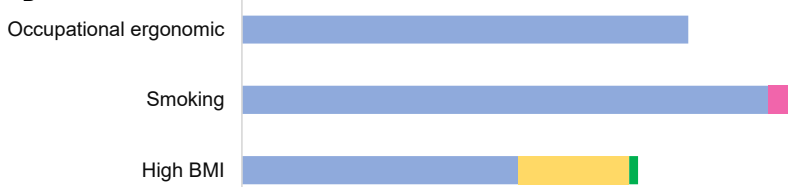

Impaired kidney function

Others ${ }^{*}$

0

100

200

DALY per 100,00

- LBP $\backsim \mathrm{RA}=\mathrm{OA} \backsim$ Gout

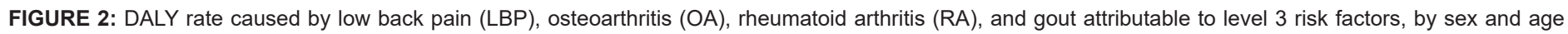

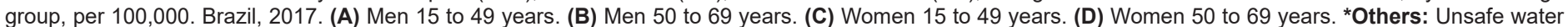

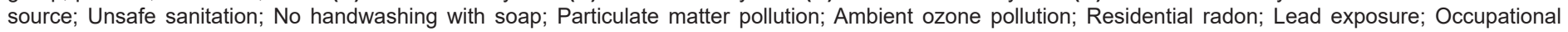

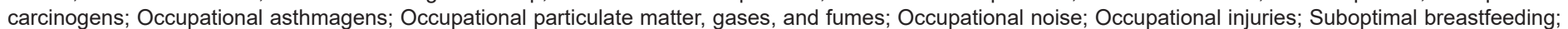

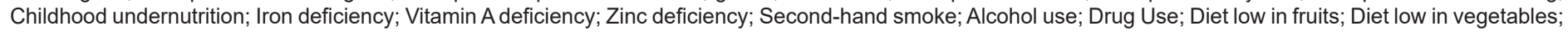

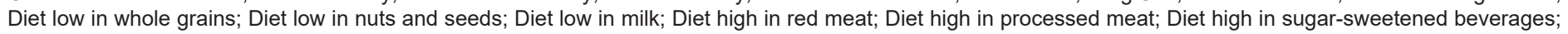

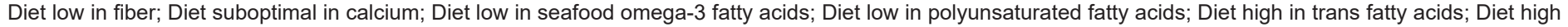

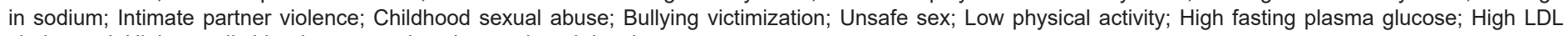
cholesterol; High systolic blood pressure; Low bone mineral density.

burden attributable to risk factors, because it was not associated with the risk factors assessed by the GBD study. The multifactorial nature of MSK disorders has been extensively documented ${ }^{1,7}$. Ergonomic risks and individual characteristics and lifestyles affect musculoskeletal function ${ }^{20}$. The proportion of DALY attributable to occupational ergonomic risk factors in this study was not surprising ${ }^{21-24}$. Statistical analyses adjusted for age, sex, level of education and duration of employment emphasized the impact of moderate to intense force application and repetitive work-related gestures on inflammatory and degenerative musculoskeletal 
processes $^{25}$. Mechanical loading of soft tissues in response to these types of operations will be enhanced if workplace design, furniture and equipment selection do not fit the characteristics of individuals working in the setting ${ }^{24,26}$. Such ergonomic problems explain awkward postures adopted by operators while manipulating work-related items ${ }^{22}$, or the excessive force required to operate machinery and equipment, for instance ${ }^{27}$. The increased BMI reflects unbalanced diets and smoking, both of which are common behaviors in the contemporary modus vivend $i^{7}$.

The evidence on environmental/occupational risk factors is relevant considering the magnitude of prevalence of LBP. It is known that LBP is experienced by approximately 50 to $80 \%$ of individuals at some point in $\operatorname{life}^{28}$, and it is the current leading cause of disability in countries where vital statistics are available, regardless of per capita income. YLD due to LBP increased 54\% worldwide, from 1990 to 2015 . These trends are partly explained by demographic changes, given the likelihood of suffering from LBP increases with age ${ }^{2,29}$.

Smoking ranked first among risk factors for LBP in the 50-69-year women group. Chemicals in tobacco products cause vasoconstriction. In turn, decreased blood perfusion leads to intervertebral disc tissue malnutrition and development of degenerative intervertebral disk lesions responsible for low back symptoms. Tobacco chemicals are also a risk factor for osteoporosis and related $\mathrm{LBP}^{30}$. In the literature, gender-related disparities may be explained by exposure differences. In industries involving tasks associated with intense physical force use in awkward postures, the exposed population comprises primarily males ${ }^{24}$. Different from men of similar age, women aged 50-69 years are exposed to the effects of decreased estrogen levels on musculoskeletal tissues. There is evidence of severe intervertebral disk degeneration in post-menopausal women ${ }^{31,32}$.

The association between high BMI and LBP can be explained by different mechanisms. Spinal overload in response to body weight gain puts localized pressure on tissues of this region. Obesity is associated with increased cytokine production and resulting activation of inflammatory processes. Obesity-related chronic systemic inflammation may be a cause of $\mathrm{LBP}^{33}$.

RA is a systemic autoimmune condition with rising prevalence and age-standardized incidence rates ${ }^{34}$. Genetic and hormone factors, stress, obesity, infections and other factors have all been incriminated. However, smoking is widely recognized as the primary risk factor for this morbidity ${ }^{35}$, as shown in this study. Chemicals in cigarettes act as inflammatory mediators and play a significant role in development of aggressive joint damage. Although not gender-related, this effect is thought to be more pronounced in men $^{36}$. Age and gender differentials were not observed in the RA burden. Regarding the occupational risks, they were not analyzed in GBD as already mentioned.

OA manifests through a variety of clinical presentations and affects primarily women aged 50-69 years. The contribution of genetic factors, dietary habits and obesity to symptom development is widely recognized ${ }^{37}$. Despite the multifactorial nature of OA, obesity is thought to be a key factor in the development and progression of hip, knee, ankle, foot, and shoulder osteoarthritic lesions. Osteoarticular overload produced by excess body weight increases the risk of $\mathrm{OA}$ and bone fractures. Aside from the mechanical factor, inflammatory mediators produced by fat tissues are harmful to joint cartilages ${ }^{38}$.

The burden of gout was attributed to high BMI. Similar findings have been reported in the GBD study comparing the global burden of this morbidity across different countries between 1990 and $2017^{39}$. High BMI was a contributing factor to rising gout rates among men and women. Potential relations between excessive dietary intake of red meats, seafood, and sweet foods and beverages, in countries with higher gout prevalence have been suggested. These foods are associated with higher levels of urate, typical of gout. Reduced dyslipidemia and serum urate levels in individuals who lost weight in response to changes in proportions of dietary macronutrients support this hypothesis ${ }^{39}$.

MSK disorders affect a fifth of the Brazilian adult population. They are more prevalent in the population of the South region, among women, increased with advancing age and individuals with a low education level. Besides the risk factors addressed in this study, gender, age, education, insufficient physical activity, and the presence of morbidities can be associated with MSK disorders ${ }^{40}$. Age is one of the most common risk factors. Longer life expectancy and population growth may be related to an increase in the burden of MSK disorders ${ }^{41}$.

The prevalence of MSK disorders is expected to increase significantly over the next decades. This will likely translate into higher burden of disease ${ }^{41}$, especially in low- and middle-income countries ${ }^{7}$. MSK disorders share etiologic and aggravation risk factors with other chronic noncommunicable diseases (NCD). Ideal body weight, practice of regular physical activity, balanced diet, smoking cessation, and other healthy behaviors, need to be emphasized in programs aimed to tackle chronic NCD in Brazil.

However, recent results indicate the need to improve interventions in this area, because, despite the decrease in the prevalence of smoking since $1990^{42}, 10 \%$ of Brazilian adults are still smokers ${ }^{43}$. Regarding overweight, the prevalence in the Brazilian adult population is higher among men $(56.5 \%$ of men and $49.1 \%$ of women). The obesity prevalence is continuing to increase both in the Brazilian state capitals and the Federal District, indicating the need for revision of policies ${ }^{44}$.

Surprisingly, MSK disorders are not regarded as a priority and related environmental/occupational risk factors are not included in the national chronic NCD-tackling agenda ${ }^{45}$. Prevention approach to reduce MSK disorders should include healthy behaviors and ergonomically suitable work environment ${ }^{46}$. Also, timely access to the health system to identify and treat early and good-quality information on what can be done to prevent or effectively manage MSK disorders is needed ${ }^{47}$.

This study has limitations, which should be considered. First, the proportion of DALY not attributable to the risk factors assessed by the GBD Study is high. NP and other MSK disorders were 
not included in this analysis, because they were not associated with risk factors examined in the GBD study. The GBD results do not allow for comparison of all risk factors related to DALY for each type of MSK addressed. As noted for RA, OA, and gout, only behavioral risk factors are included. The literature associates exposure to ergonomic risk factors and RA, but the GBD data do not allow for further elaboration of these hypotheses ${ }^{48,49}$. This limit has consequences for interpretations of the results obtained in the present study. GBD data do not allow exploring the burden of neck pain, for example, attributable to occupational risks as shown in a study ${ }^{50}$. Furthermore, the entire burden of OA is not assessed, because only hip and knee OA is included in GBD Study. LPB estimates are based on self-reported data, which can result on bias. Another limitation is that the results depend on the quality and quantity of the input data for the models. Moreover, the $95 \%$ uncertainty interval of the estimates is large in several cases, and results should be taken with caution. Despite these limitations, this study advanced in analyzing the LBP, RA, OA, and gout attributable to risks factors for Brazil. To our knowledge, the results on the LBP burden attributable to ergonomic risks is pioneering in Brazil. The prevalence of these diseases in Brazil has been widely documented ${ }^{9-12,51}$. However, this is the first national study to compare LBP, RA, OA, and gout burden attributable to risk factors. This study revealed occupational ergonomic factors as an important risk factor to LBP.

The findings of this study emphasized the need for better health and work sector policies, and suggested MSK disorders and occupational risk factors should be included in the agenda to address chronic NCD. Occupational surveillance measures are indicated in order to prevent LBP. Actions to decrease smoking and surveillance weight gain are warranted to decrease the burden of LBP, RA and OA, and gout, respectively. These actions will be more effective if age and sex differentials are considered in the planning. Determination of the burden of LBP, RA, OA, and gout, per Brazilian region and a continuing investigation of the burden attributable to environmental/occupational risks for NP, OA, RA, and gout are warranted. Furthermore, this study suggests the need for different gender approaches regarding the formulation of professional policies and actions aimed to prevent MSK disorders and respective subcategories.

\section{FINANCIAL SUPPORT}

Coordenação de Aperfeiçoamento de Pessoal de Nível Superior (CAPES finance code 001). Conselho Nacional de Desenvolvimento Científico e Tecnológico (CNPq 312175/2017-5).

\section{AUTHORS' CONTRIBUTION}

JW: Design of study, Bibliographic study, Research and analysis of data, Interpretation of data, Writing manuscript, Critical review of the content, Final approval of the version to be submitted; EBF: Design of study, Interpretation of data, Critical review of content, Final approval of the version to be submitted; AAA: Design of study, Bibliographic study, Interpretation of data, Writing manuscript, Critical review of the content, Final approval of the version to be submitted.

\section{CONFLICT OF INTEREST}

The authors declare they have no conflict of interest.

\section{ORCID}

Juliana Wolf: 0000-0002-7284-7169

Elisabeth Barboza França: 0000-0001-6984-0233

Ada Ávila Assunção: 0000-0003-2123-0422

\section{REFERENCES}

1. Punnett L, Wegman DH. Work-related musculoskeletal disorders: the epidemiologic evidence and the debate. J Electromyogr Kinesiol. 2004;14(1):13-23.

2. Ferreira GD, Silva MC, Rombaldi AJ, Wrege ED, Siqueira FV, Hallal PC. Prevalência de dor nas costas e fatores associados em adultos do Sul do Brasil: estudo de base populacional. Rev Bras Fisioter. 2011;15(1):316.

3. Hoy D, Brooks P, Blyth F, Buchbinder R. The Epidemiology of low back pain. Best Pract Res Clin Rheumatol. 2010;2(6):769-81.

4. Herin F, Vézina M, Thaon I, Soulat JM, Paris C, ESTEV group. Predictive risk factors for chronic regional and multisite musculoskeletal pain: a 5-year prospective study in a working population. Pain. 2014;155(5):937-43.

5. Ministério da Fazenda (MF). Secretaria de Previdência, Empresa de Tecnologia e Informações da Previdência. Anuário Estatístico da Previdência Social - AEPS 2017. Brasília: MF/DATAPREV; 2018. 908 p.

6. GBD 2017 Disease and Injury Incidence and Prevalence Collaborators. Global, regional, and national incidence, prevalence, and years lived with disability for 354 diseases and injuries for 195 countries and territories, 1990-2017: a systematic analysis for the Global Burden of Disease Study 2017. Lancet. 2018;392:1789-858.

7. Jin Z, Wang D, Zhang H, Liang J, Feng X, Zhao J, et al. Incidence trend of five common musculoskeletal disorders from 1990 to 2017 at the global, regional and national level: results from the global burden of disease study 2017. Ann Rheum Dis. 2020;79(8)1014-22.

8. Sebbag E, Felten R, Sagez F, Sibilia J, Devilliers H, Arnaud L. The world-wide burden of musculoskeletal diseases: a systematic analysis of the World Health Organization Burden of Diseases Database. Ann Rheum Dis. 2019;78:844-8.

9. Fernandes RCP, Carvalho FM, Assunção AA. Prevalence of musculoskeletal disorders among plastics industry workers. Cad Saude Publica. 2011;27(1):78-86.

10. BarbosaREC,AssunçãoAA,Araújo TM.Distúrbiosmusculoesqueléticos em trabalhadores do setor saúde de Belo Horizonte, Minas Gerais, Brasil. Cad Saude Publica. 2012;28(8):1569-80.

11. Ceballos AGC, Santos GB. Factors associated with musculoskeletal pain among teachers: sociodemographics aspects, general health and well-being at work. Rev Bras Epidemiol. 2015;18(3):702-15.

12. Assunção AA, Abreu MNS. Factor associated with self-reported work-related musculoskeletal disorders in Brazilian adults. Rev Saude Publica. 2017;51 suppl 1:10s.

13. IHME. Global Burden of Disease (GBD). [Internet]. Seattle: University of Washington; 2020 [cited 2020 Jan 17]. Available from: http://www. healthdata.org/gbd. 
14. IHME. GBD Data Visualizations. [Internet]. Seattle: University of Washington; 2020 [cited 2020 Mai 09]. Available from: http://www. healthdata.org/gbd/data-visualizations.

15. GBD 2017 Risk Factor Collaborators. Global, regional, and national comparative risk assessment of 84 behavioural, environmental and occupational, and metabolic risks or clusters of risks for 195 countries and territories, 1990-2017: a systematic analysis for the Global Burden of Disease Study 2017. Supplementary appendix 1. Lancet. 2018;92:1923-94.

16. IHME. Global Burden of Disease Study 2017 (GBD 2017): Data Input Sources Tool [Internet]. Seattle: University of Washington; 2020 [cited 2020 Jan 27]. Available from: http://ghdx.healthdata.org/gbd-2017/datainput-sources.

17. Gelman A, Greenland S. Are confidence intervals better termed "uncertainty intervals"? BMJ. 2019 Sep 10;366:15381.

18. GBD 2017 Causes of death Collaborators. Global, regional, and national age-sex-specific mortality for 282 causes of death in 195 countries and territories, 1980-2017: a systematic analysis for the Global Burden of Disease Study 2017. Supplementary appendix 1. Lancet. 2018;392:173688.

19. GBD 2017 DALYS and HALE Collaborators. Global, regional, and national disability-adjusted life-years (DALYs) for 359 diseases and injuries and healthy life expectancy (HALE) for 195 countries and territories, 1990-2017: a systematic analysis for the Global Burden of Disease Study 2017. Supplementary appendix 1. Lancet. 2018;392:1859922.

20. Micheletti JK, Bláfoss R, Sundstrup E, Bay H, Pastre CM, Andersen LL. Association between lifestyle and musculoskeletal pain: cross-sectional study among 10,000 adults from the general working population. BMC Musculoskelet Disord. 2019;20(1):609.

21. Cantley LF, Taiwo OA, Galusha D, Barbour R, Slade MD, TessierSherman B, et al. Effect of systematic ergonomic hazard identification and control implementation on musculoskeletal disorder and injury risk. Scand J Work Environ Health. 2014 Jan;40(1):57-65.

22. Caicoya M, Delclos GL. Work demands and musculoskeletal disorders from the Spanish National Survey. Occup Med (Lond). 2010;60(6):44750.

23. Tak S, Calvert GM. The estimated national burden of physical ergonomic hazards among US workers. Am J Ind Med. 2011;54(5):395-404.

24. Jäger M, Jordan C, Theilmeier A, Wortmann N, Kuhn S, Nienhaus A, et al. Lumbar-load analysis of manual patient-handling activities for biomechanical overload prevention among healthcare workers. Ann Occup Hyg. 2013;57(4):528-44.

25. Gallagher S, Heberger JR. Examining the interaction of force and repetition on musculoskeletal disorder risk: a systematic literature review. Hum factors. 2013;55(1):108-24.

26. Vandergrift JL, Gold JE, Hanlon A, Punnett L. Physical and psychosocial ergonomic risk factors for low back pain in automobile manufacturing workers. Occup Environ Med. 2012;69(1):29-34.

27. Mayer J, Kraus T, Ochsmann E. Longitudinal evidence for the association between work-related physical exposures and neck and/ or shoulder complaints: a systematic review. Int Arch Occup Environ Health. 2012;85(6):587-603.

28. McBeth J, Jones K. Epidemiology of chronic musculoskeletal pain. Best Pract Res Clin Rheumatol. 2007;21(3):403-25.

29. Hartvigsen J, Hancock MJ, Kongsted A, Louw Q, Ferreira ML, Genevay $\mathrm{S}$, et al. What low back pain is and why we need to pay attention. Lancet. 2018;391(10137):2356-67.
30. Shiri R, Karppinen J, Leino-Arjas P, Solovieva S, Viikari-Juntura E. The association between smoking and low back pain: a meta-analysis. Am J Med. 2010;123(1):87.e7-35.

31. Wu A, Dong W, Liu S, Cheung JPY, Kwan KYH, Zeng X, et al. The prevalence and years lived with disability caused by low back pain in China, 1990 to 2016: findings from the global burden of disease study 2016. Pain. 2019;160(1):237-45.

32. Kwon BK, Roffey DM, Bishop PB, Dagenais S, Wai EK. Systematic Review: Occupational Physical Activity and Low Back Pain. Occup Med (Lond). 2011;61(8):541-8.

33. Shiri R, Karppinen J, Leino-Arjas P, Solovieva S, Viikari-Juntura E. The association between obesity and low back pain: a meta-analysis. Am J Epidemiol. 2010;171(2):135-54.

34. Abate M, Vanni D, Pantalone A, Salini V. Cigarette smoking and musculoskeletal disorders. Muscles, ligaments and tendons J. 2013;3(2):63-9.

35. Safiri S, Kolahi AA, Hoy D, Smith E, Bettampadi D, Mansournia ML, et al. Global, regional and national burden of rheumatoid arthritis $1990-$ 2017: a systematic analysis of the Global Burden of Disease study 2017. Ann Rheum Dis. 2019;78(11):1463-71.

36. Lahiri M, Morgan C, Symmons DP, Bruce IN. Modifiable risk factors for RA: prevention, better than cure? Rheumatology (Oxford). 2012;51(3):499-512.

37. Bortoluzzi A, Furini, F, Scirè CA. Osteoarthritis and its managementepidemiology, nutritional aspects and environmental factors. Autoimmun Rev. 2018;17(11):1097-104.

38. Wearing SC, Hennig EM, Byrne NM, Steele JR, Hills AP. Musculoskeletal disorders associated with obesity: a biomechanical perspective. Obes Rev. 2006;7(3):239-50.

39. Xia Y, Wu Q, Wang H, Zhang S, Jiang Y, Gong T, et al. Global, regional and national burden of gout, 1990-2017: a systematic analysis of the Global Burden of Disease Study. Rheumatology (Oxford). 2019;59(7):1529-38.

40. Bezerra MAM, Hellwig N, Pinheiro GRC, Lopes CS. Prevalence of chronic musculoskeletal conditions and associated factors in Brazilian adults - National Health Survey. BMC Public Health. 2018;18:287.

41. March L, Smith EUR, Hoy DG, Cross MJ, Sanchez-Riera L, Blyth F, et al. Burden of disability due to musculoskeletal (MSK) disorders. Best Pract Res Clin Rheumatol. 2014;28(3):353-66.

42. GBD 2015 Tobacco Collaborators. Smoking prevalence and attributable disease burden in 195 countries and territories, 1990-2015: a systematic analysis from the Global Burden of Disease Study 2015. Lancet. 2017;389(10082):1885-906.

43. Malta DC, Stopa SR, Iser BPM, Bernal RTI, Claro RM, Nardi ACF. Risk and protective factors for chronic diseases by telephone survey in capitals of Brazil, Vigitel 2014. Rev Bras Epidemiol. 2015;18(Suppl 2):238-55.

44. Flores-Ortiz R, Malta DC, Velasquez-Melendez G. Adult body weight trends in 27 urban populations of Brazil from 2006 to 2016: a populationbased study. Plos one. 2019;14(3):e0213254.

45. Assunção AA, França EB. Anos de vida perdidos por DCNT atribuídos aos riscos ocupacionais no Brasil: estudo GBD 2016. Rev Saude Publica. 2020;54:28.

46. Modey GM; Brooks PM. Improving musculoskeletal health: Global issues. Best Pract Res Clin Rheumatol. 2012;26(2):237-49.

47. Woolf AD, Brooks P, Akesson K, Mody GM. Prevention of musculoskeletal conditions in the developing world. Best Pract Res Clin Rheumatol. 2008;22(4):759-72. 
48. Too CL, Muhamad NA, Ilar A, Padyukov L, Alfredsson L, Klareskog $\mathrm{L}$, et al. Occupational exposure to textile dust increases the risk of rheumatoid arthritis: results from a Malaysian population-based casecontrol study. Ann Rheum Dis. 2016;75:997-1002.

49. Parks CG, Meyer A, Beane Freeman LE, Hofmann JN, Sandler DP. Farming tasks and the development of rheumatoid arthritis in the agricultural health study. Occup Environ Med. 2019;76(4):243-9.
50. Simões MRL, Assunção AA, Medeiros AM. Dor musculoesquelética em motoristas e cobradores de ônibus da Região Metropolitana de Belo Horizonte, Brasil. Cien Saude Colet. 2018;23(5):1363-74.

51. Malta DC, Oliveira MMD, Andrade SSCA, Caiaffa WT, Souza MFM, Bernal RTI. Factors associated with chronic back pain in adults in Brazil. Rev Saude Publica. 2017;51(suppl 1):9s. 